\title{
Intracavitary pulmonary aspergilloma: endoscopic aspects
}

Aspergiloma pulmonar intracavitário: aspectos endoscópicos

\author{
Evelise Lima ${ }^{1}$, André Louis Lobo Nagy ${ }^{1}$, Rodrigo Abensur Athanazio ${ }^{2}$
}

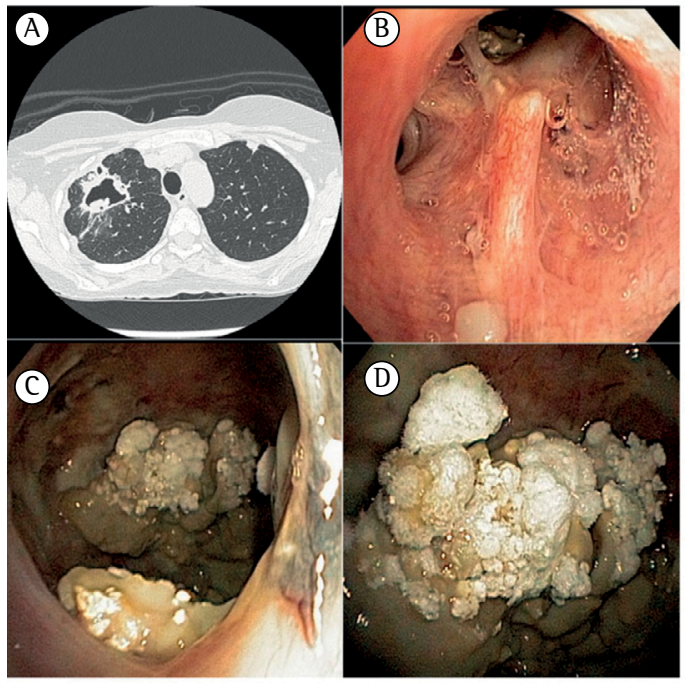

Figure 1 - $\ln$ A, CT scan of the chest showing an intracavitary mass suggestive of fungus ball. In B, right upper lobe orifice showing destruction of the architecture of the apical segment. In C and D, a whitish intracavitary mass with areas of necrosis and an endoscopic appearance suggestive of fungal infection.

A 49-year-old female patient presented with a diagnosis of parathyroid carcinoma that had been made in 2007. She underwent surgical treatment and chemotherapy.

Five years later, the patient developed cough and fever. A CT scan of the chest revealed a thin-walled cavitary lesion in the right upper lobe. Bronchoscopy with transbronchial biopsy confirmed the diagnosis of pulmonary metastasis. After radioablation therapy, the patient developed cavitary sequelae that were followed up radiologically.

A follow-up CT scan of the chest obtained in 2013 revealed an increase in the size of the cavitary lesion, thickening of its walls, and an intracavitary image suggestive of fungus ball.

The patient underwent a second bronchoscopy, which showed structural change in the apical segment of the right upper lobe, with a large cavity containing an extensive, whitish, exophytic, irregular mass.

Biopsies and bronchoalveolar lavage were performed that confirmed the diagnosis of infection with Aspergillus fumigatus.

The patient was started on oral antifungal treatment and began outpatient follow-up treatment.

Aspergillosis is a multifaceted disease whose clinical manifestations (allergic, saprophytic, and invasive forms) are determined by the host immune response.

Invasive pulmonary aspergillosis has emerged as an infectious disease of high morbidity and mortality in immunocompromised patients and should be treated early. Voriconazole is recommended as the treatment of choice.

In some cases, there can be extensive destruction of the parenchymal architecture, allowing communication with the central airway and enabling endoscopic intracavitary visualization. However, this is an extremely rare finding. In the literature, case reports are limited to patients with endobronchial aspergilloma. Endoscopically, whitish necrotic masses suggest fungal infection. However, the diagnosis should be confirmed via histopathological documentation and positive culture.

\section{Recommended reading}

1. Schweer KE, Bangard C, Hekmat K, Cornely OA. Chronic pulmonary aspergillosis. Mycoses. 2014;57(5):257-70. http://dx.doi.org/10.1111/myc. 12152

2. Patterson KC, Strek ME. Diagnosis and treatment of pulmonary aspergillosis syndromes. Chest. 2014;146(5):1358-68. http://dx.doi.org/10.1378/ chest.14-0917

3. Ma JE, Yun EY, Kim YE, Lee GD, Cho YJ, Jeong YY, et al. Endobronchial aspergilloma: report of 10 cases and literature review. Yonsei Med J. 2011;52(5):787-92. http://dx.doi.org/10.3349/ymj.2011.52.5.787

1. Attending Physician. Department of Respiratory Endoscopy, Heart Institute, University of São Paulo School of Medicine Hospital das Clínicas, São Paulo, Brazil.

2. Attending Physician. Department of Pulmonology, Heart Institute, University of São Paulo School of Medicine Hospital das Clínicas, São Paulo, Brazil. 